\title{
A rare simultaneous coexistence of epithelioid gastrointestinal stromal tumors and schwannoma in the stomach: a case report
}

Yuxin Li, Yongliang Teng, Xiaofei Wei, Zhuang Tian, Yuqing Cao, Xiaona Liu and Xiumei Duan*

\begin{abstract}
Background: Gastrointestinal stromal tumors (GISTs), a type of mesenchymal tumor in the gastrointestinal tract, are believed to be closely associated with PDGFRA and C-KIT mutations. Schwannoma in the stomach, which is an unusual location, is a rare disorder. The simultaneous occurrence of the two diseases is rarer than metachronous occurrences, and its pathological characteristics have not been reported to date. We present a case report on a patient with simultaneous coexistence of gastric schwannoma and GISTs.

Case presentation: A 39-year-old female visited our hospital complaining of intermittent abdominal pain for the previous 3 months. CT revealed a $3.4 \mathrm{~cm}$ slight homogeneous enhancement in the lesser curvature of the stomach; the mass was nodular soft tissue, which was removed by radical surgery. Two solid tumors with different volumes were located in the stomach. Histologically and immunohistochemically different, the larger tumor consisted of spindle cells surrounded by a peripheral lymphoid cuff, and was positive for S-100. The larger tumor was therefore classified as a gastric schwannoma. The smaller tumor was composed of medium-sized round, oval cells with amphiphilic granular cytoplasm; vacuolization was also observed. The tumor cells were positive for DOG1 and sporadically positive for CD34 and CD117. Hence, the smaller tumor was diagnosed as epithelioid GISTs. Sanger sequencing revealed that the GIST tumor cells contained a deletion mutation (c.2527_2538 del12,843-846del4), which was located in exon 18 of PDGFRA.
\end{abstract}

Conclusion: GISTs combined with gastric schwannoma are a considerably rare subgroup of gastric tumors. Related clinical research is comparatively weak, and the mechanism remains unknown. We reviewed related articles to provide knowledge to improve the correct identification, diagnosis and management of patients with gastric cancer. All pathologists involved in the diagnosis and clinicians involved in the treatment should be aware of this new kind of disease pattern to improve their understanding of the disease.

Keywords: GISTs, Gastric schwannoma, Case report, PDGFRA, Stomach

\section{Background}

Gastrointestinal stromal tumors (GISTs), leiomyoma or leiomyosarcoma and gastric schwannoma are tumors of the mesenchymal tissue originating in the stomach; of these, GISTs are the most common mesenchymal tumors in the gastrointestinal tract, accounting for approximately $80 \%[1,2]$. GISTs can occur in any part of the digestive

\footnotetext{
* Correspondence: xmduan@jlu.edu.cn

Department of Pathology, the First Hospital of Jilin University, Changchun, China
}

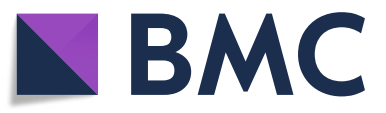

(c) The Author(s). 2019 Open Access This article is distributed under the terms of the Creative Commons Attribution 4.0 International License (http://creativecommons.org/licenses/by/4.0/), which permits unrestricted use, distribution, and reproduction in any medium, provided you give appropriate credit to the original author(s) and the source, provide a link to the Creative Commons license, and indicate if changes were made. The Creative Commons Public Domain Dedication waiver (http://creativecommons.org/publicdomain/zero/1.0/) applies to the data made available in this article, unless otherwise stated.

tract, but the most common location is the stomach $(50 \% \sim 60 \%)$, followed by the small intestine, colorectal area and esophagus; they rarely occur in the mesenteric, retinal and abdominal cavities [1, 3-7]. GISTs are mainly divided into categories based on morphology: typically a spindle pattern, an epithelioid pattern or a mixed pattern, among which the spindle pattern is the most frequent. Preoperative diagnosis of GISTs is usually established on the basis of computerized tomography (CT) of the abdomen and pelvis or magnetic resonance imaging (MRI). 
Pathologic diagnosis of GISTs is based on identification of a mesenchymal neoplasm with spindle cell or epithelioid histology. Common histologic features of GISTs include spindle cells with sclerosis matrix and epithelioid cytology in gastric GISTs [4]. Immunohistochemistry is also a significant method for diagnosing GISTs. CD117, DOG1, CD34, Ki-67 and succinate dehydrogenase B (SDHB) are recommended. With the development of precision medicine, molecular identification is becoming more important in the diagnosis of GISTs. GISTs commonly harbor oncogene mutations in the KIT tyrosine kinase, which is a target for the kinase inhibitor imatinib. A subset of GISTs, however, contains mutations in the homologous kinase platelet-derived growth factor receptor alpha (PDGFRA), and the most common of these mutations is resistant to imatinib [3, 4, 7]. GISTs have been reported to coexist with a variety of neoplasms; the percentage of such cases has ranged from 2.95 to $43 \%$ [8], but the coexistence of GISTs and gastric schwannoma is rarely found.

\section{Case presentation}

\section{Clinical history}

A 39-year-old female visited our hospital complaining of intermittent abdominal pain for the previous 3 months. The clinical doctor gave her a physical examination: the abdomen was flat, and the abdominal mass was not touched. The clinical diagnosis was stomach swelling and digestive tract hemorrhage. An upper gastrointestinal endoscopy revealed a swollen mass in the gastric antrum and angle. CT revealed a $3.4 \mathrm{~cm}$ slight homogeneous enhancement, which was nodular soft tissue in the lesser curvature of the stomach; the body of stomach was poorly filled; the mucosae and serosa were smooth; the definite margin of the tumor was surrounded by fat; and no enlarged lymph node after the abdominal cavity and peritoneum was found. Her disease was diagnosed as gastric tumors. She received laparoscopic gastric resection for gastric lesions.

\section{Pathological findings}

According to gross examination, two different solid tumors with different volumes were found in the stomach, measuring $4.3 \mathrm{~cm}^{*} 3.3 \mathrm{~cm} * 2.7 \mathrm{~cm}$ and $2.6 \mathrm{~cm}^{*} 2 \mathrm{~cm}^{*} 1.8$ $\mathrm{cm}$. Histologically and immunohistochemically, the larger tumor consisted of spindle cells surrounded by a peripheral lymphoid cuff (Fig. 1b), which was arranged mainly in small bundles or in a woven pattern (Fig. 1a). The tumor cells were positive for S-100 (Fig. 2b) and negative for CD117, DOG1 (Fig. 2a, c), CD34, Desmin, smooth muscle actin (SMA) and H-caldesmon (data not shown); the Ki-67 labeling index of the cancer cells was less than $5 \%$ (data not shown). The larger tumor was therefore classified as a gastric schwannoma. The smaller one was composed of medium-sized round, oval cells with amphiphilic cytoplasm that was granular, and vacuolization was observed. The tumor was lamellar, at which the boundary between tumor and normal cells was well demarcated (Fig. 1c, d). The tumor cells were positive for DOG1 (Fig. 2f), sporadically positive for CD34 (data not shown) and CD117 (Fig. 2d), and negative for S-100 (Fig. 2e), Desmin, SMA, H-caldesmon, and CK-pan (data not shown). Hence, the smaller tumor was diagnosed with epithelioid GISTs. A total of less than 5 mitoses per 50 high power fields (with a 40 objective) were assessed in most cellular neoplastic areas. The estimation of recurrence risk or death of the GISTs in our case could be considered low risk, depending on tumor size and the mitotic index (Table 1).

Why did GISTs and gastric schwannoma occur at the same time? Did they have a relationship to the gene mutation? To answer these questions, exons 9, 11, 13, 17 of C-KIT and exons 12 and 18 of PDGFRA were analyzed by PCR and Sanger sequencing both in GISTs
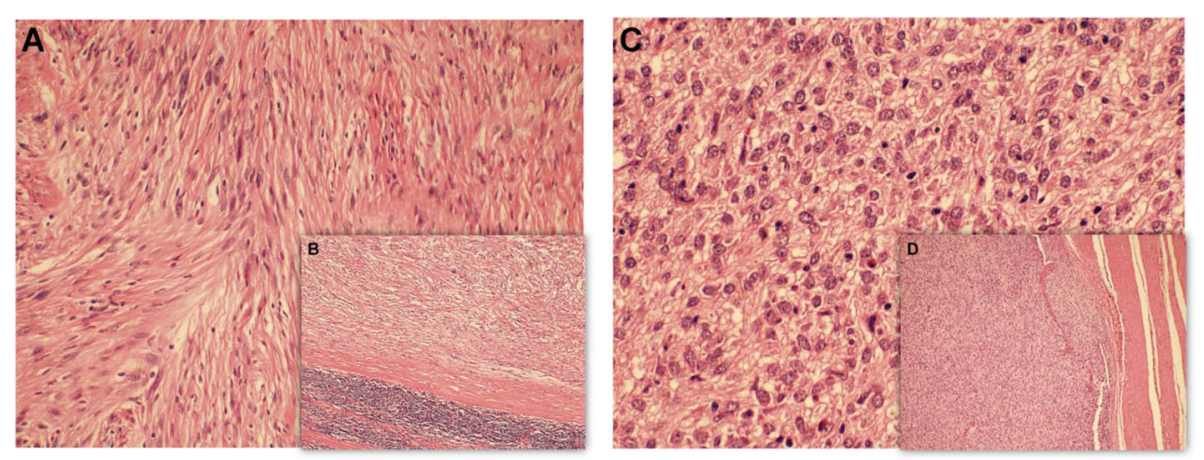

Fig. 1 Histopathological findings. Hematoxylin-eosin(HE). a $\times 20$ objective, $\mathbf{b} \times 10$ objective) Gastric schwannoma. Spindle cells surrounded by a peripheral lymphoid cuff, which was arranged mainly in small bundles or in a woven pattern. $\mathbf{c} \times 40$ objective, $\mathbf{d} \times 10$ objective) GISTs. Mediumsized round, oval cells with amphiphilic cytoplasm that was granular, and vacuolization was observed.The tumor was lamellar, at which the boundary between tumor and normal cells was well demarcated 


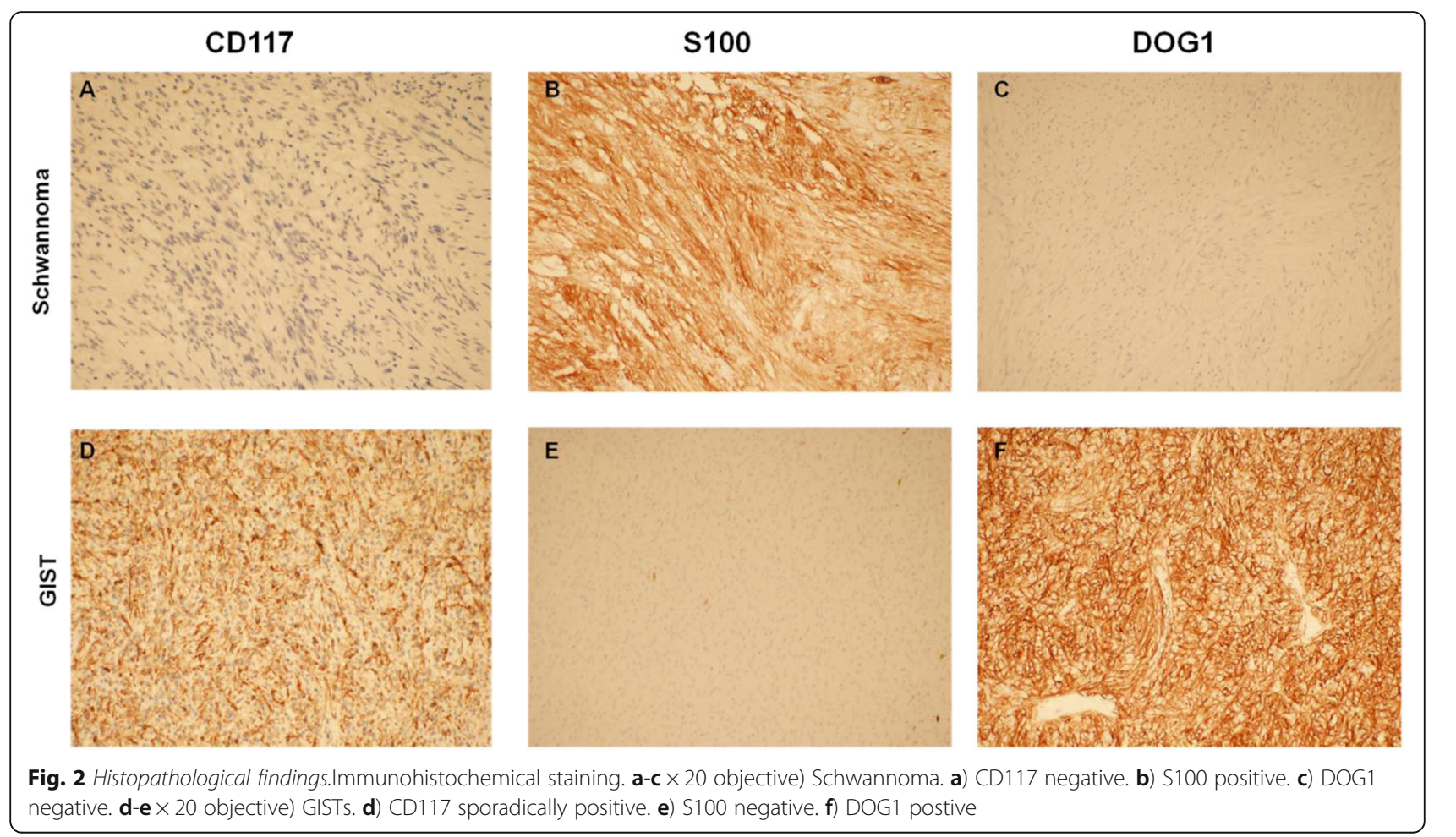

and gastric schwannoma. The tumor cells of GISTs contained a deletion mutation (c.2527_2538 del12,843846del4), which was located in exon 18 of PDGFRA (Fig. 3). However, no gene mutation was detected in gastric schwannoma.

\section{Discussion}

We present a rare case of the simultaneous occurrence of GISTs and gastric schwannoma. Various studies so far have focused on GISTs, so we have unilateral precise recognition in the pathogenesis, diagnosis, and treatment of GIST. However, studies on the mechanisms of the simultaneous occurrence of these two types of tumors are infrequent, as described below.

Table 1 Estimation of the recurrence risk or death linked to the disease in localized and resectable GISTs depending on tumor size and the mitotic index [9]

\begin{tabular}{lll}
\hline Risk & $\begin{array}{l}\text { Maximum } \\
\text { diameter }(\mathrm{cm})\end{array}$ & $\begin{array}{l}\text { Mitotic index } \\
\text { (per 50 fields) }\end{array}$ \\
\hline Very low risk & $<2$ & $<5$ \\
Low risk & $2-5$ & $<5$ \\
Intermediate risk & $<5$ & $6-10$ \\
High risk & $5-10$ & $<5$ \\
& $>5$ & $>5$ \\
& $>10$ & All \\
& All & $>10$ \\
\hline
\end{tabular}

We believe the most interesting thing about this case is that these two tumors occurred in the stomach at the same time. Highlighting of reports on the specific mechanism of GISTs and gastric schwannoma that occur simultaneously in the stomach is important, due to the remarkably peculiar coexistence, has not been systematically demonstrated. In general, the occurrence of gastric schwannoma alone is already rare. A study by Tao [2] is one of a small number of articles that have a large amount of data on gastric schwannoma. The study showed that 267 patients had gastric GISTs during the same time period-that is, approximately 9 cases of gastric GISTs were observed for each case of gastric schwannoma. Nevertheless, Yang's case report [10] is the only study that reported the simultaneous occurrence of GISTs and gastric schwannoma. Unfortunately, they did not perform further research on patients with special morphological features, and currently, no systematic study concerning the detection of genetic mutations has been published yet.

However, knowledge about the coexistence of GISTs and other gastrointestinal tumors is increasing, which can provide some inspiration for understanding the new coexisting pattern in our case. Vassos et al. summed various studies on these coexistent diseases and found that the reported frequencies of second malignancies in GIST patients ranged from 2.95 to $43 \%$. Carcinomas of the gastrointestinal tract were the most frequent neoplasms associated with concomitant GISTs; other types 


\section{Mutation in PDGFRA exon18}

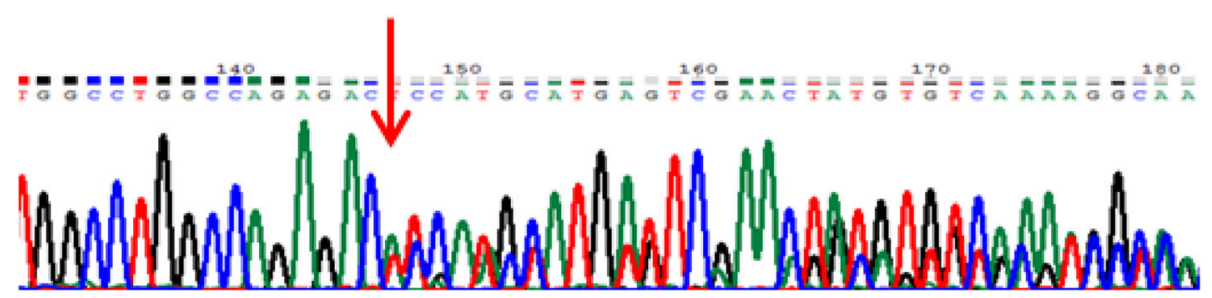

Fig. 3 Exon 18 of PDGFRA is deletion mutation type (c.2527_2538 del12,843-846del4) in GISTs

include lymphoma, lung or adrenocortical adenoma. The studies also mentioned that most synchronous GISTs were very low/low risk, and this feature also matched the characteristics of our case [8]. Regrettably, little is known about the etiology and pathogenesis of this coexistence. Simple coincidence could be the obvious explanation, but various hypotheses have been proposed. Some carcinogens, such as N-methyl-N-nitro-N-nitrosoguanidine, are involved in the development of neoplasms associated with concomitant GISTs in experiments [8]. Theoretically, genetic mutations or combined genetic deregulation could also be associated with the pathogenesis of gastric synchronous tumors. Separately, for our case, in addition to the aforementioned possible causes, we believe the same starting factors of different mesenchymal tumors are involved in the pathogenesis of these two entities because both gastric stromal tumor and gastric schwannoma originate in the mesenchyme.

However, there are many excellent reviews on GISTs alone in the literature dealing with the basic concepts of the pathogenesis of GISTs, which is related to C-KIT and PDGFRA, and the activation of its downstream pathways has been confirmed. The KIT receptor activating mutations occur in $60-96 \%$ of GISTs [3-7, 11-13]. Although the mutation rate of PDGFRA is significantly lower than that of CKIT (3.5\% 7.2\%), it is also one of the mechanisms of the pathogenesis of GISTs $[6,14]$. In the analysis of PDGFRA gene mutations, approximately $95 \%$ were observed in the stomach, omentum and peritoneum. The most common mutation is located in exon 18, where p.D842V accounts for 60 to 65\%, and a few occur in exons 12 and 14 [6, 1115]. In our case report, the gene mutation of GISTs is the deletion mutation of exon 18 in PDGFRA, which involves deletion of codons 843-846. An interesting report on the investigation of PDGFRA mutations in GISTs was described by Christopher et al. [14]. DIMH842-845/ IMH843-846 accounted for $14.9 \%$ of the total mutation in PDGFRA. Since codons $843-845$ were flanked by aspartic acid (D842 and D846), these two mutations in the protein sequence were equivalent. This is supported by Li Yanyan et al. [13], who performed gene mutation analysis on 827 patients. Their findings revealed that only two cases of
DIMH 842-845 and one case of IMH 843-846 were identified as deletion mutations. Therefore, the genetic mutation in our case is relatively scarce in GISTs. A recent review by Corless [14] revealed that deletion mutations of $842-845$ and 843-846 of exon 18 in PDGFRA were sensitive to imatinib. In contrast, mutations in the schwannoma of the stomach are infrequent.

The gastric schwannoma and GISTs are from the same mesenchymal tissue of the stomach, which is easily confused and misdiagnosed. Tao et al. [2] showed that the correct preoperative diagnosis rate of schwannoma was only $3.3 \%$, and 21 of 30 patients were misdiagnosed with GISTs. In summary, the identification of these two tumors is of particular importance. The diagnosis of schwannoma is based on immunohistochemical staining of S-100 and vimentin positive and negative results of CD117 and CD34, whereas GISTs are usually positive for CD117, DOG1 and CD34, but S-100 is mostly negative $[2,4,11,13]$. In addition, CD117 positivity can be found in approximately 95\% of C-KIT mutations, but approximately $40 \%$ of PDGFRA mutations are weakly positive or negative for CD117 $[4,14,16]$. The positive expression rate of DOG1 in GISTs is up to $94.8 \%$, and it has a high sensitivity of $89 \%$, and its positive rate in epithelioid GISTs is higher than that in CD117 [4, 12, 17].

\section{Conclusion}

We describe the first known case report on the coexistence of gastric schwannoma with epithelioid GISTs using genetics, immunochemistry and histology at our institute. These rare and unique coexistent tumors could possibly be misdiagnosed preoperatively, especially when the diagnosis is based solely on CT without histological and immunohistochemical analyses. Furthermore, we have observed this phenomenon from the perspective of genetics, which could provide a potential kind of pathogenesis and a new pattern of co-occurrence for neoplasms associated with concomitant GISTs. The concurrence of other malignancies with GISTs raises discussion about a potential common origin and carcinogenetic mechanisms in these 
distinct tumor types; thus, a case series study would be needed. Finally, we expect that our case report would contribute to the recognition of morphological and biological characteristics of these coexistent diseases, which support the precise diagnosis and correct interpretation to avoid inappropriate under- or overtreatment of the patients in the future.

\section{Abbreviations}

CT: Computerized tomography; GISTs: Gastrointestinal stromal tumors; MRI: Magnetic resonance imaging; PDGFRA: Platelet-derived growth factor receptor alpha; SDHB: Succinate dehydrogenase B; SMA: Smooth muscle actin

\section{Acknowledgments}

Not applicable.

\section{Authors' contributions}

YuL: conception and writing of manuscript. YuL, XiW, ZhT and XiD: PDGFRA and C-KIT mutation analysis. YuL and YuC: collection of clinical data. YuL and YuT: pathological diagnosis and immunohistochemical analyses. YuC, XiL and XiD: revision of manuscript. All authors read and approved the final manuscript prior to submission.

\section{Funding}

Not applicable.

\section{Availability of data and materials}

The datasets used and/or analyzed during the current study are available from the corresponding author upon reasonable request.

\section{Ethics approval and consent to participate}

Not applicable.

\section{Consent for publication}

Written informed consent was obtained from the patient for the publication of this case report.

\section{Competing interests}

The authors declare that they have no competing interests.

Received: 14 May 2019 Accepted: 4 October 2019

Published online: 23 October 2019

\section{References}

1. Søreide K, Sandvik OM, Søreide JA, Giljaca V, Jureckova A, et al. Global epidemiology of gastrointestinal stromal tumours (GIST): a systematic review of population-based cohort studies. Cancer Epidemiol. 2016;40:39-46.

2. Tao K, Chang W, Zhao E, Deng R, Gao J, et al. Clinicopathologic features of gastric Schwannoma. Medicine (Baltimore). 2015;94(45):e1970.

3. Gopie P, Mei L, Faber AC, Grossman SR, Smith SC, et al. Classification of gastrointestinal stromal tumor syndromes. Endocr Relat Cancer. 2018; 25(2):R49-58.

4. Markku M, Jerzy L. Gastrointestinal stromal tumors. Gastroenterol Clin N Am. 2013:42(2):399-415.

5. Liang J, Wu YL, Chen BJ, Zhang W, Tanaka Y, et al. The C-KIT receptormediated signal transduction and tumor-related diseases. Int J Biol Sci. 2013;9(5):413-43.

6. Li Y, Gao J, Tian Y, Li J, Shen L. Interpretation of 827 cases of gastrointestinal stromal tumor C-KIT or PDGFRA gene mutation spectrum and its relationship with clinicopathological features. Chin J Gastrointest Surg. 2015; 18(4):332-7.

7. Michael S, Emmanouel D, Charikleia S, Panagiotis S, Electra P, et al. Gastrointestinal stromal tumor. World J Surg Oncol. 2009;7:61.

8. Vassos N, Agaimy A, Hohenberger W, Croner RS. Coexistence of gastrointestinal stromal tumours (GIST) and malignant neoplasms of different origin: prognostic implications. Int J Surg. 2014;12(5):371-7.
9. Fletcher CD, Berman JJ, Corless C, Gorstein F, Lasota J, et al. Diagnosis of gastrointestinal stromal tumors: a consensus approach. Hum Pathol. 2002; 33(5):459-65.

10. Yang $Y$, Yang J, Tang L, Shi X, Wu S, et al. Missed diagnosis of gastrointestinal stromal tumor with gastric schwannomas: a case report. Clinical Misdiagnosis Mistherapy. 2017;30(28):20-1.

11. Lasota J, Miettinen M. Clinical significance of oncogenic KIT and PDGFRA mutations in gastrointestinal stromal tumours. Histopathol. 2008;53(3):245-66.

12. Miettinen M, Lasota J. Gastrointestinal stromal tumors: review on morphology, molecular pathology, prognosis, and differential diagnosis. Arch Pathol Lab Med. 2006;130(10):1466-78.

13. Li J, Cai H, Wang W. Relationship between c-KIT and PDGFRA gene mutations in gastrointestinal stromal tumors and clinicopathological features, immunophenotype and prognosis. J Clin Exp Pathol. 2018. 34(8):834-9.

14. Corless CL, Schroeder A, Griffith D, Diana G, Ajia T, et al. PDGFRA mutations in gastrointestinal stromal tumors: frequency, spectrum and in vitro sensitivity to imatinib. J Clin Oncol. 2005;23(23):5357-64.

15. Lasota J, Dansonka-Mieszkowska A, Sobin LH, Miettinen M. A great majority of GISTs with PDGFRA mutations represent gastric tumors of low or no malignant potential. Lab Invest. 2004;84:874-83.

16. Debiec-Rychter M, Wasag B, Stul M, De WI, Van OA, et al. Gastrointestinal stromal tumours (GISTs) negativefor KIT (CD117 antigen)immunoreactivity. J Pathol. 2004;202:430-8.

17. Lopes LF, West RB, Bacchi LM, Van RM, Bacchi CE. DOG1 for the diagnosis of gastrointestinal stromal tumor (GIST): comparison between 2 different antibodies. Appl Immunohistochem Mol Morphol. 2010;18(4):333-7.

\section{Publisher's Note}

Springer Nature remains neutral with regard to jurisdictional claims in published maps and institutional affiliations.

\section{Ready to submit your research? Choose BMC and benefit from:}

- fast, convenient online submission

- thorough peer review by experienced researchers in your field

- rapid publication on acceptance

- support for research data, including large and complex data types

- gold Open Access which fosters wider collaboration and increased citations

- maximum visibility for your research: over $100 \mathrm{M}$ website views per year

At BMC, research is always in progress.

Learn more biomedcentral.com/submissions 